\title{
SUBCORTICAL EFFERENT PROJECTIONS OF THE MEDIAL GENICULATE NUCLEUS MEDIATE EMOTIONAL RESPONSES CONDITIONED TO ACOUSTIC STIMULI ${ }^{1}$
}

\author{
JOSEPH E. LEDOUX, ${ }^{2}$ AKIRA SAKAGUCHI, AND DONALD J. REIS \\ Laboratory of Neurobiology, Cornell University Medical College, New York, New York 10021 \\ Received June 21, 1983; Revised August 24, 1983; Accepted September 7, 1983
}

\begin{abstract}
The purpose of this study was to identify the afferent link in the neural pathway which mediates emotional responses coupled to auditory stimuli. We evaluated whether autonomic and behavioral responses elicited by acoustic conditioned emotional stimuli are based on afferent information derived from the auditory cortex or from the auditory thalamic relay station, the medial geniculate nucleus (MG), in rats. The rat auditory cortex was defined through anterograde neuroanatomical tracing studies involving the injection of HRP into MG. Lesions were then placed in the auditory cortex or in MG. After 10 to 20 days the rats were subjected to classical fear conditioning trials involving the pairing of a pure tone with electric footshock. Changes in mean arterial pressure and heart rate and the duration of immobilization ("freezing") and drink suppression elicited by presentation during extinction trials (no footshock) of the acoustic conditioned emotional stimulus were measured. Auditory cortex lesions did not affect the magnitude of the mean arterial pressure or heart rate conditioned responses nor the duration of freezing or drink suppression. In contrast, lesions of MG suppressed the magnitude of both the autonomic and somatomotor (behavioral) conditioned emotional responses but did not affect either autonomic or somatic responses elicited by the footshock unconditioned stimulus. Lesions of the inferior colliculus, the primary source of afferent input to $\mathrm{MG}$, replicated the effects of $M G$ lesions. These findings demonstrate that lesions of $\mathrm{MG}$ and lower auditory centers, but not lesions of the auditory cortex, block autonomic and behavioral conditioned emotional responses coupled to acoustic stimuli and indicate that subcortical rather than cortical efferents of $\mathrm{MG}$ sustain these responses. Our concurrent observation that MG projects to several subcortical areas (central and lateral amygdala; caudate-putamen; ventromedial hypothalamus) involved in emotional behavior and autonomic function suggests hypotheses concerning subsequent links in this emotional processing pathway.
\end{abstract}

An important issue in psychobiology concerns the neural mechanisms which underlie the emotional interpretation of environmental stimuli. How does afferent information acquire emotional coloration as sensory signals are transferred from station to station in the brain?

Classic neurology suggests that afferent information reaching the major sensory nuclei of the dorsal thalamus is relayed by thalamocortical pathways directly to neocortical receiving areas. From there, the signals are transmitted, by way of association zones, to paleo- and subcortical regions, particularly to regions of the "limbic system." Through corticolimbic projections, to areas such

\footnotetext{
${ }^{1}$ This work was supported by Public Health Service Grant HL18974. We express our gratitude to Drs. David A. Ruggiero and Theresa Milner for consultation in the neuroanatomical studies.

${ }^{2}$ To whom correspondence should be addressed.
}

as the amygdala and hippocampus, the emotional meaning of the sensory stimulus is evaluated and behavioral responses appropriate to this meaning are controlled (Papez, 1937; MacLean, 1949, 1970; Penfield, 1958; Geschwind, 1965; Turner et al., 1980; Gray, 1982).

The view that emotional evaluation of environmental events depends upon the neocortical analysis of sensory information is supported by anatomical observations demonstrating multisynaptic projections from each sensory receiving area of the neocortex to limbic areas (Whitlock and Nauta, 1956; Jones and Powell, 1970; van Hoesen et al., 1972; Herzog and van Hoesen, 1976; Turner et al., 1980). Moreover, interruption of these multisynaptic pathways disrupts behavioral responses conditioned to the emotional meaning of complex (i.e., highly discriminated) visual (Mishkin, 1956, 1978; Downer, 1961; Horel and Keating, 1969; Jones and Mish- 
kin, 1972) and auditory (Meyer and Woolsey, 1952; Diamond and Neff, 1957; Thompson, 1960; Kelly, 1974) stimuli.

A curious and interesting feature of emotional processing is that it can be dissociated from the perceptual content of the eliciting stimulus. This observation has long been a tenet of psychoanalysis but has only recently found acceptance in the mainstream of experimental psychology. Thus, studies in humans and experimental animals have demonstrated that the affective evaluation of environmental events is often based on primitive sensory features too crude to support stimulus identification (see Zajonc, 1980; LeDoux, 1984) and actually precedes perceptual recognition (Moreland and Zajonc, 1979). These findings raise the question of whether in test situations which do not necessitate the engagement of the complex perceptual functions characteristic of neocortical sensory systems, emotional responses might be shown to be mediated by sensory information derived from subcortical sensory stations.

Indeed, it has long been recognized that complete bilateral removal of the neocortex, which necessarily destroys the neocortical sensory receiving areas, does not block the expression of autonomic responses and emotional behaviors evoked by sensory stimulation (Bechterev, 1887; Cannon and Britton, 1925; Bard and Rioch, 1937; Macht and Bard, 1942) nor the coupling, through aversive classical conditioning, of autonomic responses to relatively simple (i.e., undiscriminated) stimulus features (Pinto-Hamuy et al., 1963; Bloch-Rojas et al., 1964; DiCara et al., 1970). While there is conflicting evidence concerning the effects of decortication on the classical conditioning of somatomotor responses (Culler and Mettler, 1934; Girden et al., 1936; Bromiley, 1948; Bloch-Rojas et al., 1964), there is no indication that this possible effect of total removal of the cortex is due to damage to sensory cortex. The effects of select ablations of sensory cortex on classically conditioned somatomotor emotional responses should be examined.

If sensory cortex is not part of the neural pathway through which at least some emotional responses conditioned to sensory stimuli are mediated, then such responses must be based on sensory signals diverging from the primary projection system in thalamic or lower subcortical regions. However, there is little information available concerning the effects of selective lesions of subcortical sensory nuclei on conditioned emotional responses.

Therefore, in the present study we have examined the role of the neocortical, thalamic, and midbrain stations of the auditory system in the mediation of emotional responses classically conditioned to a pure tone in rats. The rat auditory cortex was defined through neuroanatomical tracing studies involving the microinjection of horseradish peroxidase (HRP) into the auditory thalamic relay nucleus, the medial geniculate body (MG). Lesions were then placed in the auditory cortex, in $M G$, or in the inferior colliculus (IC), the primary source of auditory input to MG. To determine whether different afferent pathways underlie the conditioning of autonomic and somatomotor responses, both were measured in the same animal. Our results indicate that select ablation of the auditory cortex does not affect either somatomotor or autonomic responses elicited by an acoustic conditioned emotional stimulus, but that these responses are disrupted by lesions of MG and lower centers in the ascending auditory pathway. We conclude that emotional processing depends upon afferent information diverging from the auditory pathway subcortically, at the level of MG. Thus, subcortical efferents of MG appear to constitute a primary link in the pathway through which the emotional significance of auditory stimuli is evaluated.

\section{Materials and Methods}

Animals. Studies were undertaken in male SpragueDawley rats $(n=90)$ weighing 300 to $400 \mathrm{gm}$ obtained from Charles River Laboratories. Animals were individually housed in clear plastic cages with free access to water and laboratory chow. The housing area was thermally controlled (at $20^{\circ} \mathrm{C}$ ), sealed to sunlight, and maintained on a fluorescent light cycle (on at 7:00 AM, off at 7:00 PM).

\section{Surgical Procedures}

Ablation of lateral neocortex. Rats were anesthetized with halothane ( 1.5 to $2.0 \%$ in $100 \% \mathrm{O}_{2}$ ) and placed in a stereotaxic frame (Kopf). The lateral surface of one hemisphere was exposed through a limited craniotomy. The auditory neocortex, as defined by neuroanatomical tracing studies (see below), was removed bilaterally through subpial aspiration using procedures described by Myers (1971). Hemorrhaging was controlled by application of Gelfoam. The wound was kept moist with cotton pellets soaked in saline while the same procedure was performed on the other hemisphere. After completion of the surgery, the wound was cleaned and the scalp was sutured. Control animals were anesthetized and placed in the stereotaxic frame, and the cranium was exposed but not removed.

Body weight and food and water consumption were routinely checked during the first postoperative week in order to detect postsurgical complications and prevent unnecessary discomfort. Ten to 20 days were allowed for recovery after surgery before initiation of behavioral studies (see below).

Stereotaxic placement of subcortical lesions. Rats were anesthetized with halothane (see above) and placed in a stereotaxic frame (Kopf). The cranium was exposed, and a small hole was made using a dental drill. A monopolar stainless steel electrode (diameter, $200 \mu \mathrm{m}$ ) insulated with epoxy to within $200 \mu \mathrm{m}$ of the tip was lowered through an incision in the dura to the region of $\mathrm{MG}$ using stereotaxic coordinates $(2.5 \mathrm{~mm}$ anterior to the intcraural line; $3.5 \mathrm{~mm}$ lateral to the midline; $3.5 \mathrm{~mm}$ dorsal to the interaural line) taken from Paxinos and Watson (1982). Bilateral electrolytic lesions were placed in MG by passing anodal constant current $(1.0 \mathrm{~mA} ; 8$ to $15 \mathrm{sec}$ ) from a Grass Instruments DC Lesion Maker through the electrode. In other rats, bilateral lesions were placed in the IC $(0.5 \mathrm{~mm}$ anterior to interaural line; 2 $\mathrm{mm}$ lateral to midline; $6 \mathrm{~mm}$ dorsal to the interaural line) or in the region of the lateral posterior and lateral geniculate (LP-IGG) thalamic nuclei $(4.0 \mathrm{~mm}$ anterior to the interaural line; $3.5 \mathrm{~mm}$ lateral to the midline; $5.2 \mathrm{~mm}$ 
dorsal to the interaural line). Sham lesions were produced by lowering the electrode into MG but without passing current. Postoperative care was performed as described above for cortical lesions.

Chronic catheterization for cardiovascular recording. Catheters were implanted 2 to $3 \mathrm{hr}$ after classical conditioning and $24 \mathrm{hr}$ before assessment of conditioned responses (see below). Procedures for implantation and maintenance of catheters have been described in earlier publications (LeDoux et al., 1983; Sakaguchi et al., 1983). In brief, the animals were anesthetized with halothane (see above). A plastic cannula (PE 50) filled with saline containing heparin (50 units $/ \mathrm{ml}$ ) was inserted into the thoracic aorta via the left common artery, and its tip was placed at the level of the diaphragm. With the cannula fixed to the soft tissues with sutures, the free end was passed subcutaneously behind the ear to the back of the neck, brought through the skin through a stab wound, and sealed with stainless steel obturators. The neck wound was closed with sutures, and the rats were returned to their home cages. The cannulas were filled daily with $0.5 \mathrm{ml}$ of $0.9 \%$ saline containing heparin (50 units $/ \mathrm{ml}$ ).

\section{Histological procedures}

Evaluation of lesions. At the end of the experiment, rats with cortical and subcortical lesions were anesthetized with Nembutal and perfused with $10 \%$ formalin through the left ventricle of the heart. The brains were sectioned at $50 \mu \mathrm{m}$, and every fourth section was saved and stained with cresyl violet. The extent of the cortical lesions was plotted on diagrams similar to those described by Lashley (1941). Subcortical lesions were plotted on diagrams based on Jones and Leavitt (1974), Paxinos and Watson (1982), and Ottersen and Ben-Ari (1979).

Neuroanatomical tracing procedures. The anterograde HRP technique was used to identify the neocortical projection of MG. The protocol described by Ruggiero et al. (1982) was followed. In brief, the rats were anesthetized with halothane and placed in a stereotaxic frame, as described above for the lesion experiments. Twenty to $30 \mathrm{nl}$ of $1 \%$ wheat germ agglutinin-conjugated HRP (Sigma) in $0.9 \%$ saline were slowly injected through a glass micropipette $(50 \mu \mathrm{m}$ diameter tip) into $\mathrm{MG}$. The pipette was left in place for $30 \mathrm{~min}$ following completion of the injection. All injections were unilateral, with the side of injection randomly varied. Postoperative care was identical to that in other experiments.

Forty-eight hours following the injection, the animals were anesthetized with Nembutal and perfused via the left ventricle of the heart. The brain was removed and sectioned at $50 \mu \mathrm{m}$. Every fourth section was processed histochemically using the tetromethylbenzidine (TMB) procedure of Mesulam (1978). Alternate sections through the region of injection were processed using the diaminobenzidine (DAB) procedure of Graham and Karnovsky (1966). The DAB-processed and remaining alternate sections were stained using cresyl violet. The location of labeled terminals and cells was plotted with the aid of a camera lucida attached to a microscope fitted with a dark field condenser. The injection site was evaluated in DAB- and TMB-processed sections using procedures described by Mesulam (1982).

\section{Behavioral methods}

\section{Classical fear conditioning}

Rats were removed from their home cage, placed in a standard conditioning chamber enclosed by a sound at-
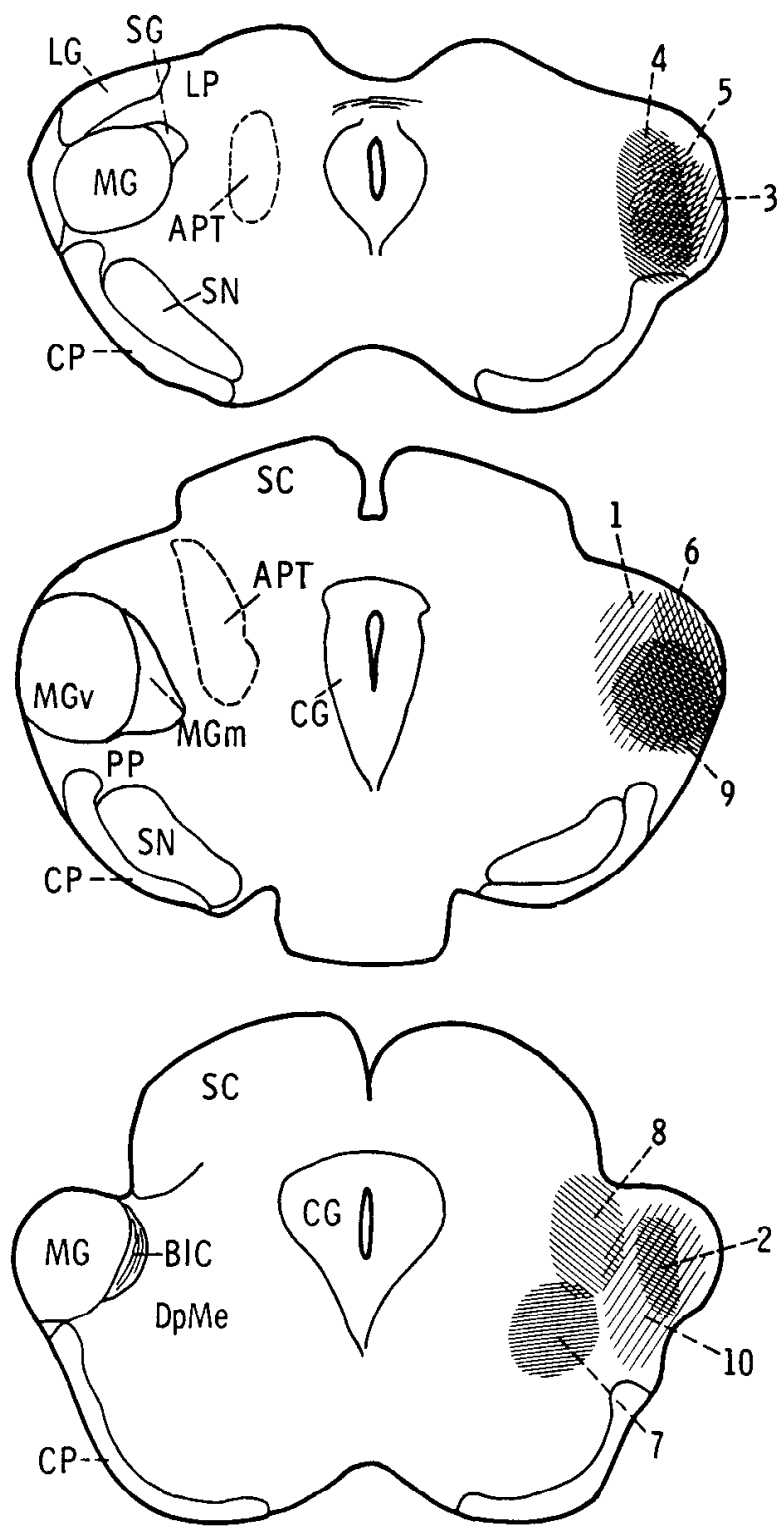

Figure 1. HRP injection sites. Wheat germ agglutinin-conjugated HRP (20 to $30 \mathrm{nl}$ ) was stereotaxically injected into the area of $\mathrm{MG}$ in 10 ràts. The location of the center of each injection (as seen in DAB-processed tissue) is depicted on the right, with anatomical detail in the region shown on the left. In eight of the cases (nos. 1 to 6,9 , and 10 ), the injection primarily involved portions of $\mathrm{MG}$. In the two remaining cases (nos. 7 and 8 ) the injections were medial to MG. $A P T$, anterior pretectal nucleus; $B I C$, brachium of the inferior colliculus; $C G$, central gray; $C P$, cerebral peduncle; $D p M e$, deep mesencephalon; $L G$, lateral geniculate nucleus; $L P$, lateral posterior thalamic nucleus; $M G$, medial geniculate body; $M G m$, medial nucleus of MG; $M G v$, ventral nucleus of MG; $P P$, peripeduncular area; $S C$, superior colliculus; $S G$, suprageniculate nucleus; $S N$, substantia nigra. 


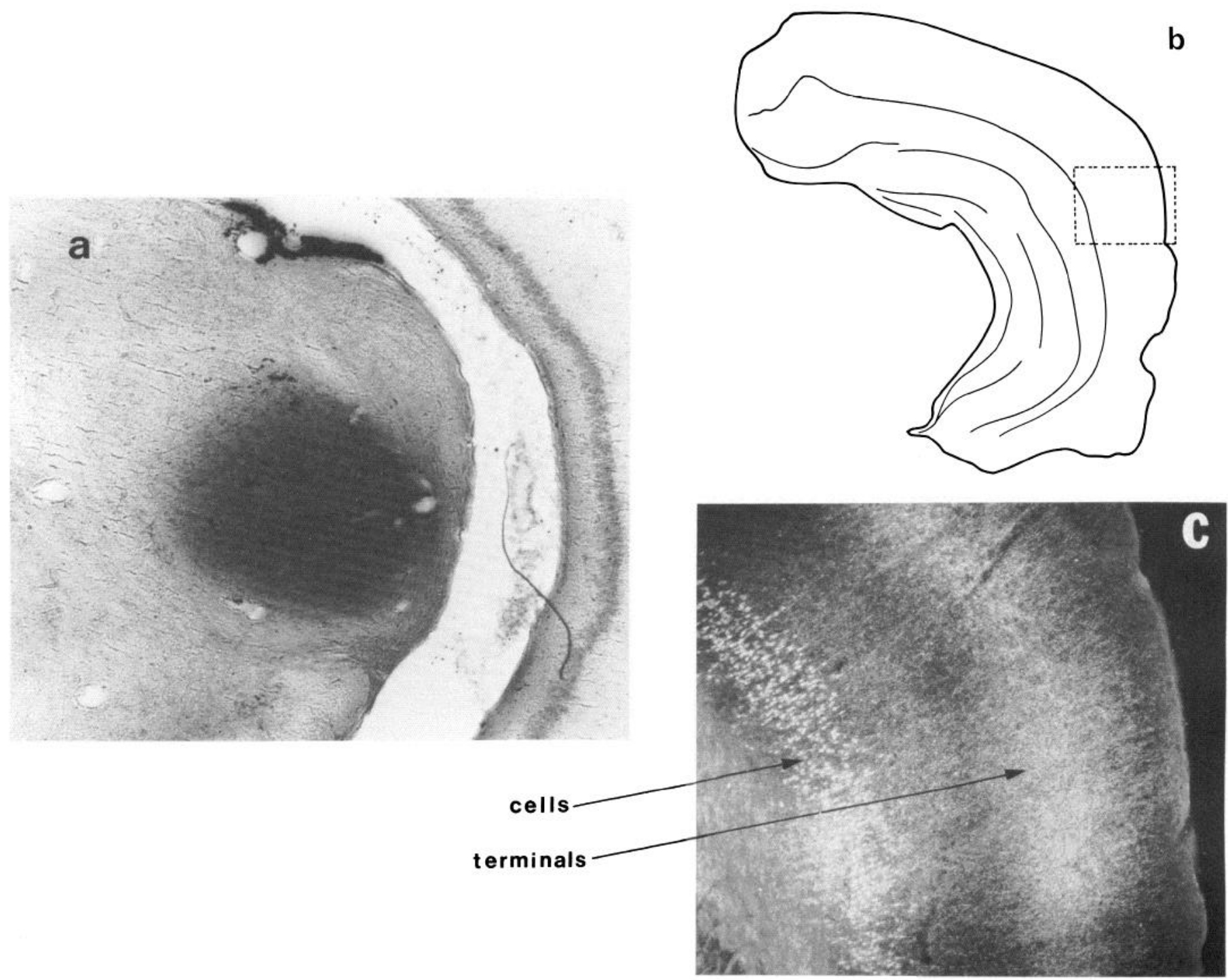

Figure 2. Anterograde and retrograde transport of HRP to neocortex. $a$, Bright field photomicrograph of an injection of HRP (approximately $20 \mathrm{nl}$ ) into MG. The section depicted was processed with DAB substrate and counterstained with cresyl violet. $b$, Drawing of a coronal section through the right hemisphere in this case. The dashed rectangle indicates the location of the photomicrograph shown in $c$. $c$, Darkfield photomicrograph showing HRP reaction product in neocortex in an unstained coronal section processed with TMB substrate from the same case. Anterograde labeling is densest in the middle cortical lamina. Retrogradely labeled cell bodies were seen in the deep lamina. These patterns of projection are characteristic of thalamocortical and corticothalamic pathways, respectively.

tenuating cubicle (Coulborun Instruments), and subjected to classical conditioning. The conditioned stimulus (conditioned emotional stimulus, CES) was a 10-sec pure tone of $800 \mathrm{~Hz}$ produced by a frequency generator, amplified to $82 \mathrm{~dB}$, and presented through a speaker mounted on the front panel of the conditioning chamber. The unconditioned stimulus (US) consisted of footshock $(2.0 \mathrm{~mA}, 0.5 \mathrm{sec})$, distributed across the grid floor of the conditioning chamber. For the first 10 trials, the CES was presented alone in order to habituate orienting responses. Over the last 30 trials, the termination of the CES was coextensive with the US. The intertrial interval averaged $150 \mathrm{sec}$ and varied between 100 and $200 \mathrm{sec}$. Stimulus presentation was controlled by a microprocessor (Apple II). Following the conditioning sessions, which lasted approximately $2 \mathrm{hr}$, the animal was returned to its home cage and allowed free access to food and water.

\section{Assessment of conditioned emotional responses}

On the day following conditioning, conditioned emotional responses (CERs) were measured. All tests involved presentation of the CES during extinction trials (no US) conducted in the animal's home cage. In this way, the contaminating effects of the US and the environmental cues (other than the CES) associated with the footshock US were eliminated, thus allowing a relatively pure assessment of the responses elicited by the CES itself. As in previous studies (LeDoux et al., 1982, 1983), we have measured both autonomic (cardiovascular) and behavioral CERs.

Cardiovascular CERs. In order to assess CES-elicited changes in mean arterial pressure (MAP) and heart rate (HR), the home cage was transferred to a partially enclosed observation area. The obturator was removed from 

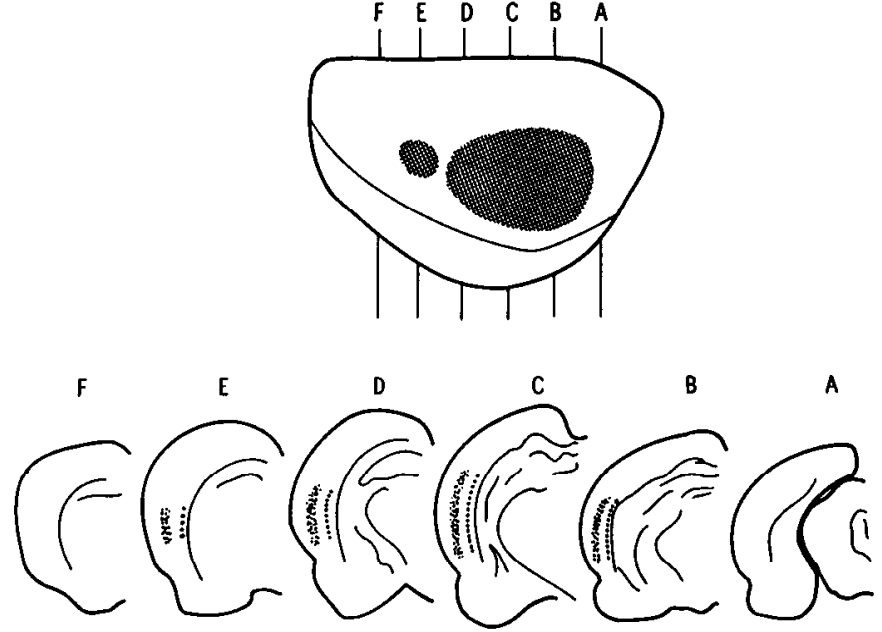

Figure 3. Neocortical distribution of efferents from MG. Injection of $\mathrm{HRP}$ into $\mathrm{MG}$ resulted in anterograde transport to two neocortical regions. These are shown in the composite drawing (top) representing the results obtained from the eight cases with injections inclusive of MG (see Fig. 1). $A$ to $F$ indicate posterior to anterior serial levels through the neocortex which are reconstructed in coronal sections (bottom), with the lamination of labeled terminals (multiple small dots) and cell bodies (single row of large dots) illustrated in each section. The drawing of the lateral surface of the left hemisphere is based on Skinner (1971).

the arterial cannula, and the free end of the cannula was attached to extension tubing by way of a stainless steel connector. The tubing was threaded through the cage top and connected to a strain gauge transducer (Statham $\mathrm{P} 23 \mathrm{Db}$ ), which was placed outside the cage at the level of the heart. The animal was carefully monitored to ensure that the tubing was not disturbed during recording.

Pulsatile and mean arterial pressure were continuously recorded from the transducer via an input coupler connected to a chart recorder (Beckman Dynagraph Type R611). HR was derived by a cardiotachometer (Beckman 9857). All data were simultaneously recorded on-line by a microcomputer (Varian V-76), which sampled MAP at a rate of $100 / \mathrm{sec}$. Incoming data from each experiment were digitized, processed, and stored on disk for subsequent analysis and display.

After $15 \mathrm{~min}$ of acclimation to test the environment, the 10 -sec CES was presented to the resting animal three times (through a speaker which was located directly above the cage) by the microcomputer. Stimulus presentation was on the same schedule as during conditioning.

Following the three trials, the computer generated a table which listed the average value (mean) and variability (standard deviation) of MAP and HR during the following time periods: $(a)$ pre-CES (averaged over 10 sec); (b) CES (averaged over $10 \mathrm{sec}$ ); (c) pre-CES average subtracted from each second of CES. In this way, the absolute value of MAP and HR during the pre-CES and CES periods and the relative change from baseline of MAP and HR during each second of the CES were computed.

Behavioral CERs. Conditioned emotional behavior was
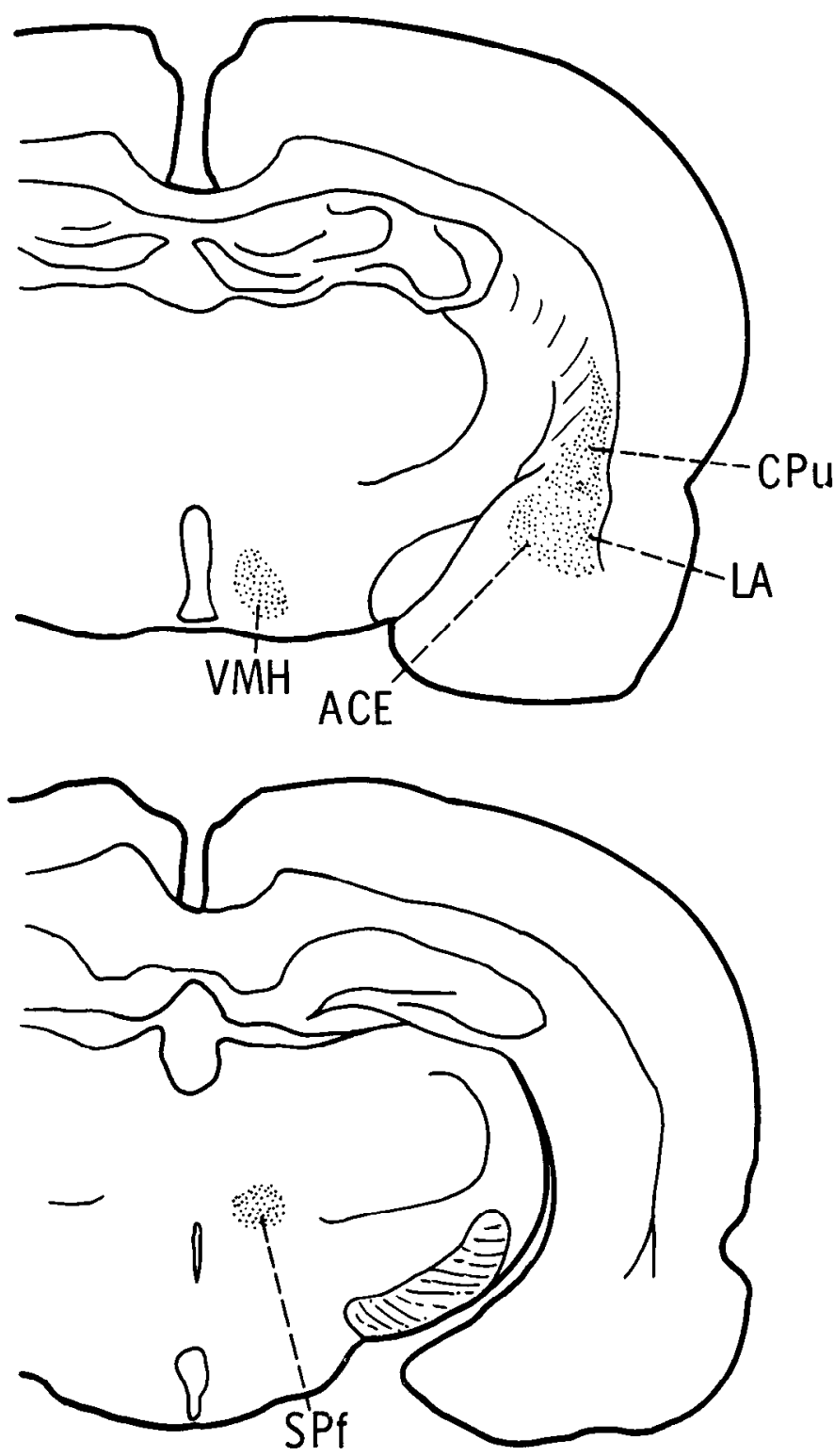

Figure 4. Subcortical efferents of MG. HRP was transported to several subcortical areas following injection of MG. Terminal fields were observed in the central $(A C E)$ and lateral $(L A)$ nuclei of the amygdala, ventromedial hypothalamic nucleus $(V M H)$, caudate-putamen $(C P u)$, and subparafascicular nucleus $(S P f)$.

measured in two ways: The duration of immobilization or "freezing" during the CES and the duration of suppression of drinking by the CES were evaluated.

The duration of freezing (Blanchard and Blanchard, 1972; Bouton and Bolles, 1980; LeDoux et al., 1982, 1983) elicited by the CES was assessed $3 \mathrm{hr}$ after the measurement of autonomic CERs. The rat was removed from its home cage and transferred to a clean cage in order to elicit exploratory activity. After a 10-sec delay, the CES was presented continuously for $120 \mathrm{sec}$ through a speaker resting over the cage, and the duration of freezing was measured using a stop watch.

Drink suppression by the CES (Leaf and Muller, 1965; LeDoux et al., 1982) was measured 3 days after asssessment of conditioned freezing. The rats were placed in 
a
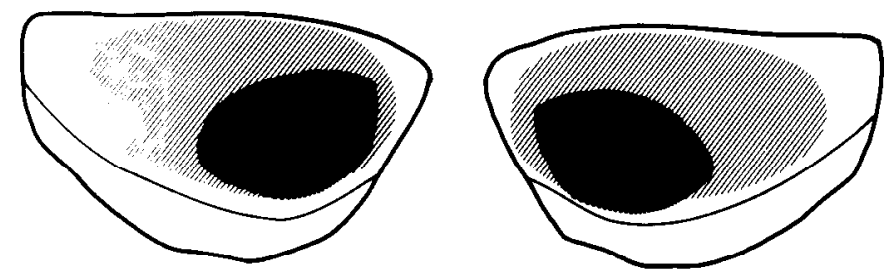

Largest lesion (ACX64)

Smallest lesion (ACX51)

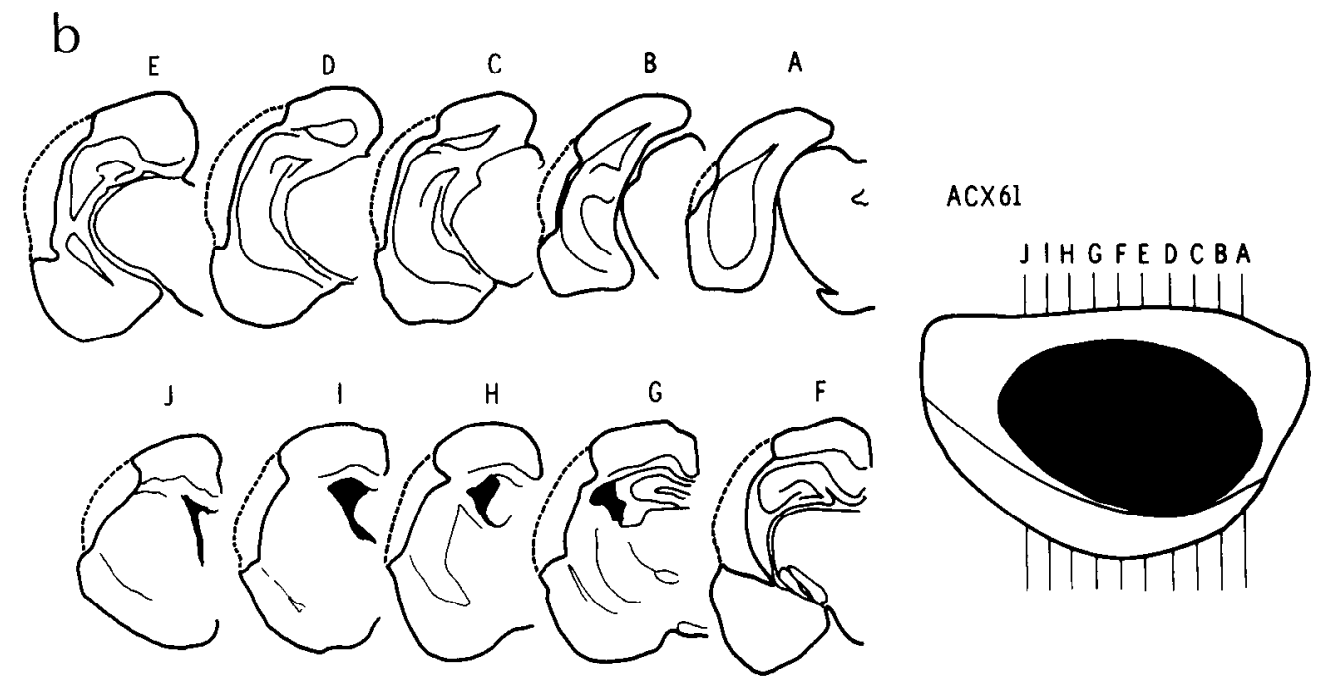

Figure 5. Lesions of the auditory cortex. Lateral neocortex was ablated bilaterally in 19 rats. The extent of damage produced by the largest and smallest cortical lesions is shown in (a). Comparison of each lesion with the neocortical distribution of MG efferents (Fig. 3) revealed that in all 14 rats the posterior terminal field was destroyed and that in five rats the anterior field was also destroyed. $b$, Serial reconstruction through one of the large lesions showing in coronal sections of the left hemisphere the extent of neocortical damage. Cortical surface in ablated regions is approximated by dashed lines. Drawings of lateral neocortex are based on Skinner (1971).

the conditioning chamber and administered an additional five trials of aversive conditioning (see above) on the day before the drink suppression test. The animal was then deprived of water overnight. The next morning, the home cage was transferred to the observation area. After a 15-min delay, a water dispenser was made available. The experimenter observed the animal's activities during this period; after $10 \mathrm{sec}$ of continuous licking were completed, the CES was presented for 120 sec. The duration of drink suppression, assessed as the time taken to resume drinking, was measured using a stop watch.

\section{Unconditioned emotional reactivity}

Unconditioned emotional reactivity was assessed by measuring reactivity to the footshock US. The animal was placed in the conditioning chamber, and the arterial catheter was connected to the transducer. After $1 \mathrm{~min}$ of exploratory activity, the animal received a single $0.5-\mathrm{sec}$, $2.0-\mathrm{mA}$ footshock. Over the subsequent $30 \mathrm{sec}$, the experimenter measured the amount of time accounted for by freezing using a stop watch. The MAP and HR were continuously recorded $5 \mathrm{sec}$ prior to the footshock and during the subsequent 5 sec.

\section{Acoustic startle response}

While the rat was resting quietly the experimenter clapped his hands and noted the presence or absence of a startle reaction consisting of a sudden and brief movement of the whole body. Fifteen seconds later, the test was repeated.

\section{Experimental design and statistical tests}

The experiments involved the assessment of the effects of auditory cortex, defined through neuroanatomical (anterograde HRP) tracing studies, MG, and IC lesions upon the changes in MAP, HR, and behavior elicited by presentation of an acoustic CES. In brief, groups of animals were subjected to surgery. These groups included rats with bilateral lesions of the auditory cortex $(n=19)$ and their sham-operated controls $(n=14)$ as well as rats with bilateral lesions of MG $(n=13)$, IC $(n=4)$, LP$\mathrm{LG}(n=6)$, or sham lesions of MG $(n=7)$. Following 10 to 20 days' recovery from surgery, the animals were subjected to classical fear conditioning, involving the association of a pure tone with electric footshock. Two or $3 \mathrm{hr}$ following conditioning, the animals were catheterized. The next morning, cardiovascular responses elicited by the CES were assessed during extinction trials 
a
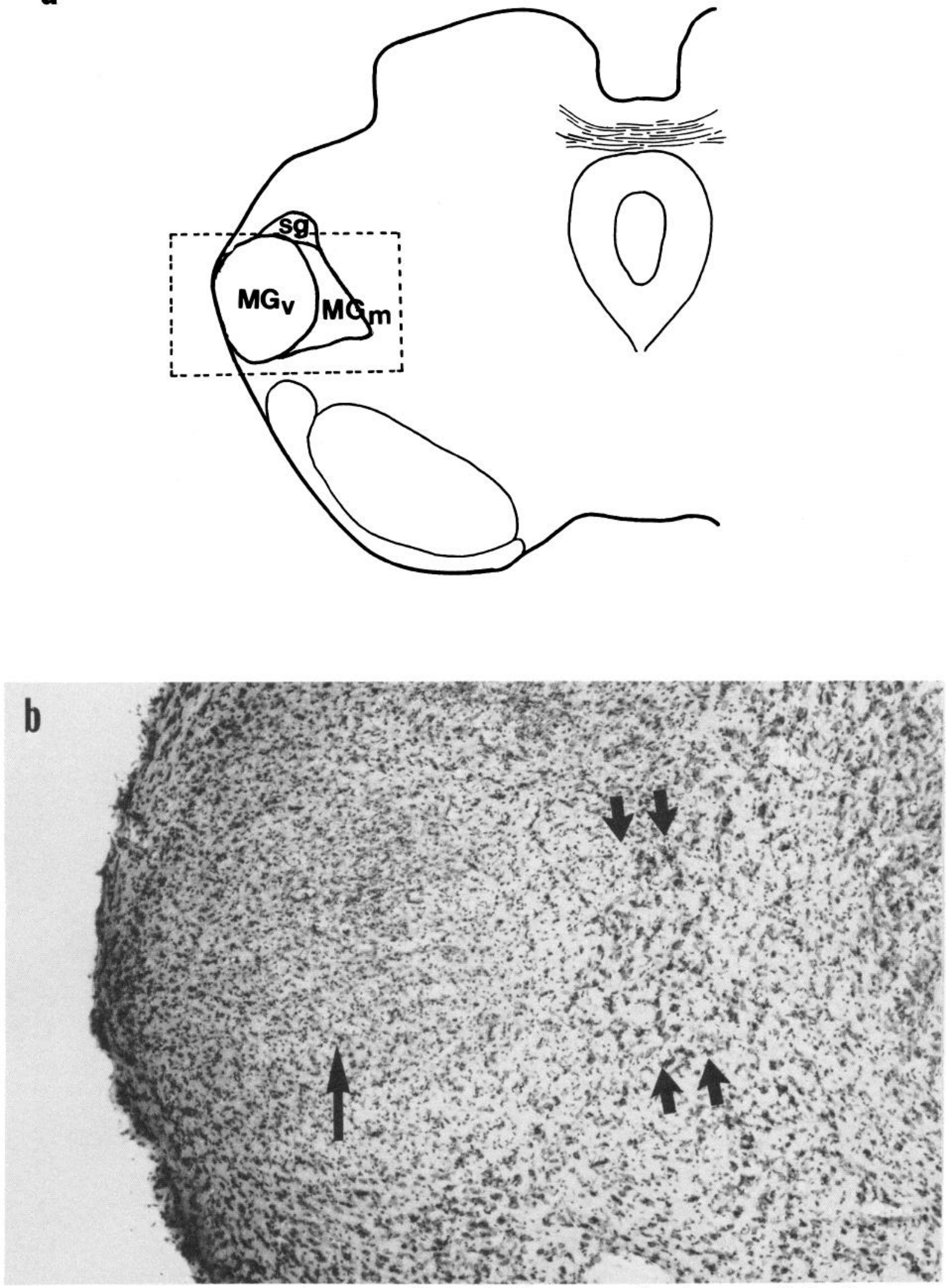

Figure 6. Retrograde cell changes in MG following ablation of auditory cortex. Complete removal of auditory cortex resulted in neuronal loss and glial proliferation in MG. Retrograde changes were most severe in the ventral nucleus of $M G\left(M G_{v}\right)$, the classic thalamocortical relay nucleus. Surviving neurons were consistently seen in the medial nucleus $\left(M G_{m}\right)$. The $d a s h e d$ rectangle in $a$ indicates the approximate location from which the photomicrograph shown in $b$ was taken. The photomicrograph shows neuronal atrophy and glial accumulation in MGv (single arrow) and surviving neurons in MGm (double arrows). 

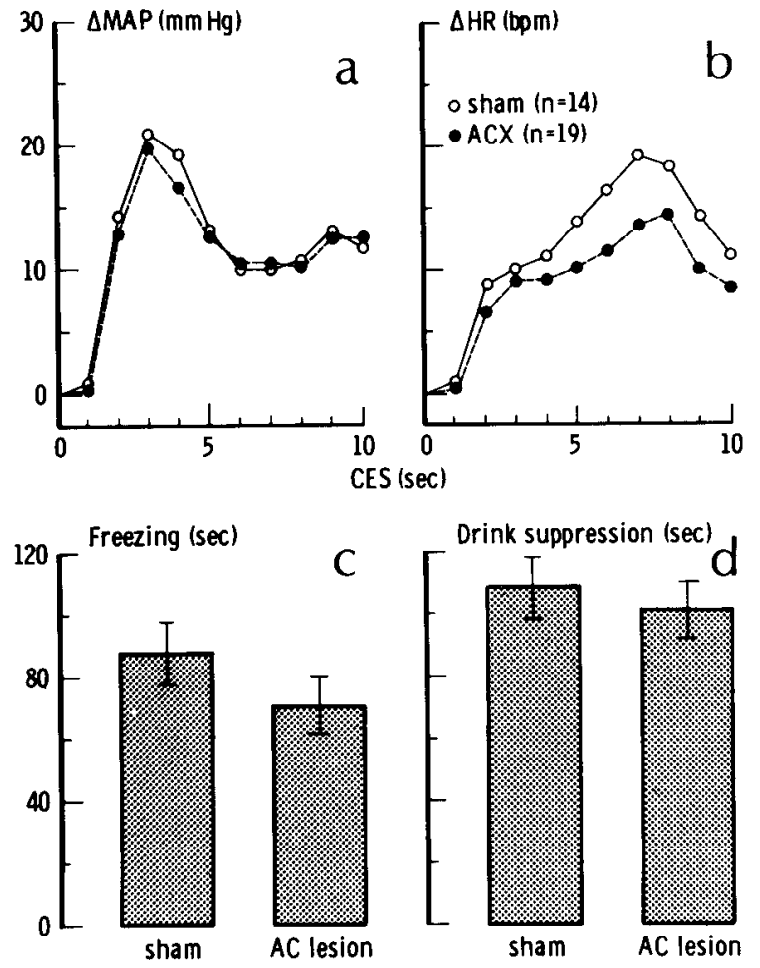

Figure 7. Effects of auditory cortex lesions on CERs. Ablations of auditory cortex (Fig. 2) did not affect autonomic or behavioral CERs. Thus, the magnitude of the pressor response $(a)$ and tachycardia $(b)$ and the extent of freezing $(c)$ and drink suppression $(d)$ elicited by the CES did not differ in lesioned and sham-operated animals. For the MAP and HR responses a two-way analysis of variance repeated measures design, with treatment (lesion versus control) as the fixed variable and every other second during the 10-sec CES as the repeated measure, was employed. The main effect of treatment was not significant for either MAP $(F(1,31)=0.05)$ or $\operatorname{HR}(F(1,30)=0.45)$. For the freezing and drink suppression responses, $t$ tests were employed and produced nonsignificant $t$ statistics (freezing: $t(31)=1.08$; drink suppression: $t(29)=0.41$.

conducted in the animal's home cage. That afternoon, CES-elicited "freezing" was measured, and acoustic startle responses were tested. Approximately 2 days later, some animals were given another five conditioning trials, deprived of water overnight, and presented with the CES while drinking the next morning in order to assess conditioned drink suppression. In a separate experiment, groups of MG $(n=6)$ and sham $(n=5)$-lesioned animals, MAP, HR, and freezing were measured in response to presentation of the footshock US alone.

All results were evaluated using analysis of variance and post-hoc tests. The particular design and tests are described in the table and figure legends along with the results.

\section{Results}

\section{Role of auditory cortex}

Anatomical distribution of geniculocortical projection. Since the auditory projection field of $M G$ is at present poorly defined in the rat, the following studies were undertaken in order to guide and evaluate lesion placement in auditory neocortex. Wheat germ agglutininconjugated HRP (20 to $30 \mathrm{nl}$ ) was injected into the area of MG in 10 rats (Fig. 1). In eight of the cases, the injection primarily involved MG. Involvement of adjacent regions was variable from case to case. In the two remaining animals the injections were medial to $\mathrm{MG}$.

Each injection involving $\mathrm{MG}$ resulted in the anterograde transport of HRP to the neocortex (Fig. 2). The anterior-posterior extension of the primary projection zone (Fig. 3) was about $4 \mathrm{~mm}$, beginning at the level of the underlying posterior superior colliculus and continuing through the anterior extent of the dorsal hippocampus, as seen in coronal sections. The ventral border was about 0.5 to $1.0 \mathrm{~mm}$ superior to the rhinal fissure throughout the anterior-posterior extension of the projection zone, except in two cases where light terminals approached the fissure. From the ventral border, terminals extended dorsally to a maximum of about $3 \mathrm{~mm}$ in the center of the region of termination.

In five of the cases (nos. 1, 3, 4, 6, and 9 in Fig. 1) with injections involving relatively anterior portions of $\mathrm{MG}$, a second, smaller, neocortical terminal zone was observed (Fig. 3). In serial sections, this region was separated from the primary area by about 0.5 to $1.0 \mathrm{~mm}$, was located at the level of the underlying posterior optic chiasm, and extended 1 to $2 \mathrm{~mm}$ rostrally. Its dorsal-ventral extent was about $1 \mathrm{~mm}$, and it was located about $1.5 \mathrm{~mm}$ superior to the rhinal fissure.

Neocortical terminals were densest in cortical layers III and IV (Figs. 2 and 3). The deep cortical layers contained areas of densely packed, retrogradely labeled cell bodies. This pattern, which is typical of thalamocortical and corticothalamic projections involving thalamic sensory nuclei, held for both the primary and secondary neocortical terminal zones.

Several subcortical areas were also labeled. Cell bodies were consistently seen in the inferior colliculus and nucleus of the lateral lemniscus in the eight cases with injections involving MG (Fig. 1). In addition, a number of nonauditory structures were labeled. Terminals were seen in the caudate-putamen, amygdala (central and lateral nuclei), hypothalamus (ventromedial nucleus), and the subparafascicular region of the thalamus (Fig. 4). Labeling of the caudate-putamen was observed in all eight cases with $\mathrm{MG}$ injections. In contrast, the amygdalar, hypothalamic, and subparafascicular regions were each labeled in four cases (nos. 1, 4, 6, and 10 in fig. 1). The amygdala was also labeled in an additional case (no. 3 ) in which the hypothalamic and subparafascicular regions were not labeled.

In the two cases with injections medial to MG, there was no evidence of anterograde transport to the neocortex nor to the subcortical areas labeled following injection of MG. These injections only resulted in anterograde transport locally.

Lesions of auditory cortex. The lateral neocortex was ablated bilaterally in 19 rats, and the extent of damage was evaluated histologically in relation to the neocortical distribution of $\mathrm{MG}$ efferents. In every case $90 \%$ or more of the primary cortical projection zone of $\mathrm{MG}$ was damaged (see Fig. 5). In five cases the lesion extended anteriorly and included both terminal zones. The extent of the largest and the smallest lesion is shown in Figure $5 a$. Figure $5 b$ represents a serial reconstruction through one of the larger lesions. 
a

01
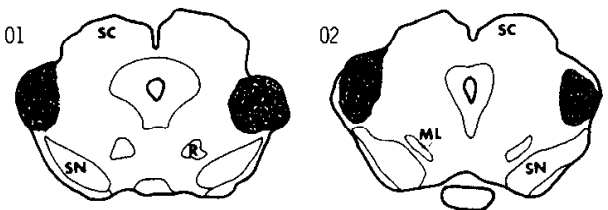

03
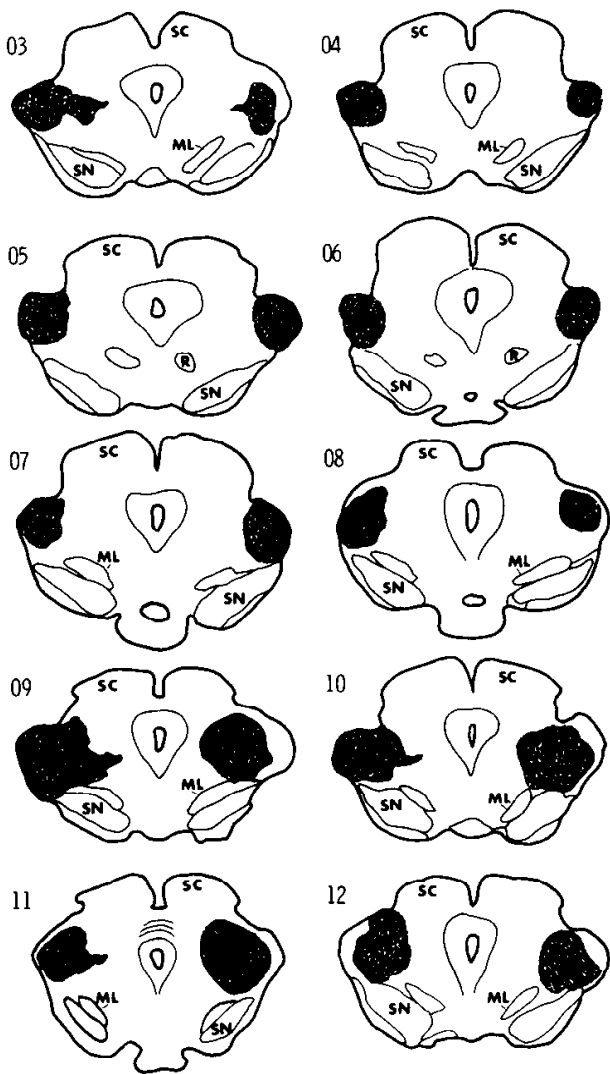

b

01

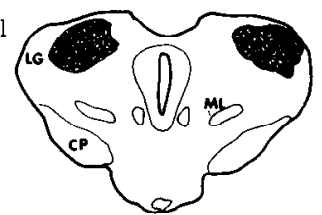

02

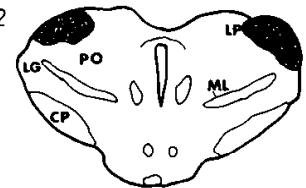

03

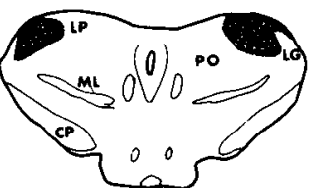

04
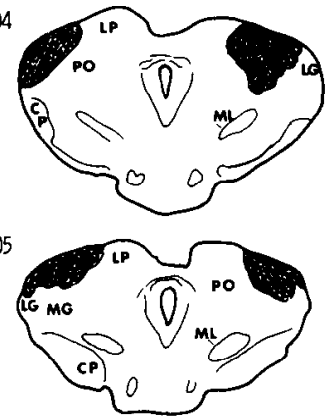

06

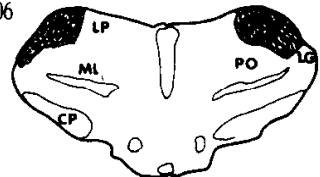

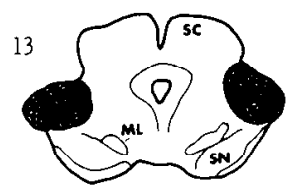

Figure 8. Lesions of MG. Lesions were placed in MG in 13 rats and in LP-LG in six rats. $a$, Coronal sections through the center of MG lesions. $b$, Coronal sections through the center of LP-LG lesions. $C P$, cerebral peduncle; $L G$, lateral geniculate nucleus; $L P$, lateral posterior nucleus; $M L$, medial lemniscus; $P O$, posterior thalamus, $R$, red nucleus; $S C$, superior colliculus; $S N$, substantia nigra.

Retrograde cell changes were evaluated in MG following the cortical lesions. As is commonly reported (Waller, 1934; Lashley, 1941; Kelly and Glazier, 1978), removal of temporoparietal cortex in rat produces neuronal loss and glia proliferation in MG. Retrograde changes were greatest in the ventral division of $M G$, the classic thalamocortical relay nucleus, and were less severe in the medial division, where surviving neurons were consistently seen (Fig. 6). The survival of medial MG neurons is consistent with Lashley's (1941) observation that, unlike other thalamic areas, retrograde degeneration of MG is always incomplete, even in cases involving massive cortical lesions.

Effects of lesions of auditory cortex on CERs. The CER in sham-operated rats $(n=14)$ consisted of an abrupt elevation of MAP and HR and the cessation of ongoing motor activity (Fig. 7). The elevation of MAP reached a maximum of $22 \pm 2 \mathrm{~mm} \mathrm{Hg} 3 \mathrm{sec}$ following the onset of the CES. The tachycardia peaked $(19 \pm 5$ beats $/ \mathrm{min})$ at the 7th sec of the CES. "Freezing" accounted for $73 \%$ of a 2-min test period, and the average amount of time taken to resume drinking following the onset of a 2-min CES was $98 \pm 12 \mathrm{sec}$.

Bilateral ablations of the auditory cortex $(n=19)$ had no consistent effect on autonomic or behavioral CERs. Thus, the lesions did not significantly modify the magnitude of the changes in MAP (Fig. 7a) or HR (Fig. 7b) or the extent of freezing (Fig. 7c) and drink suppression 


\section{a}
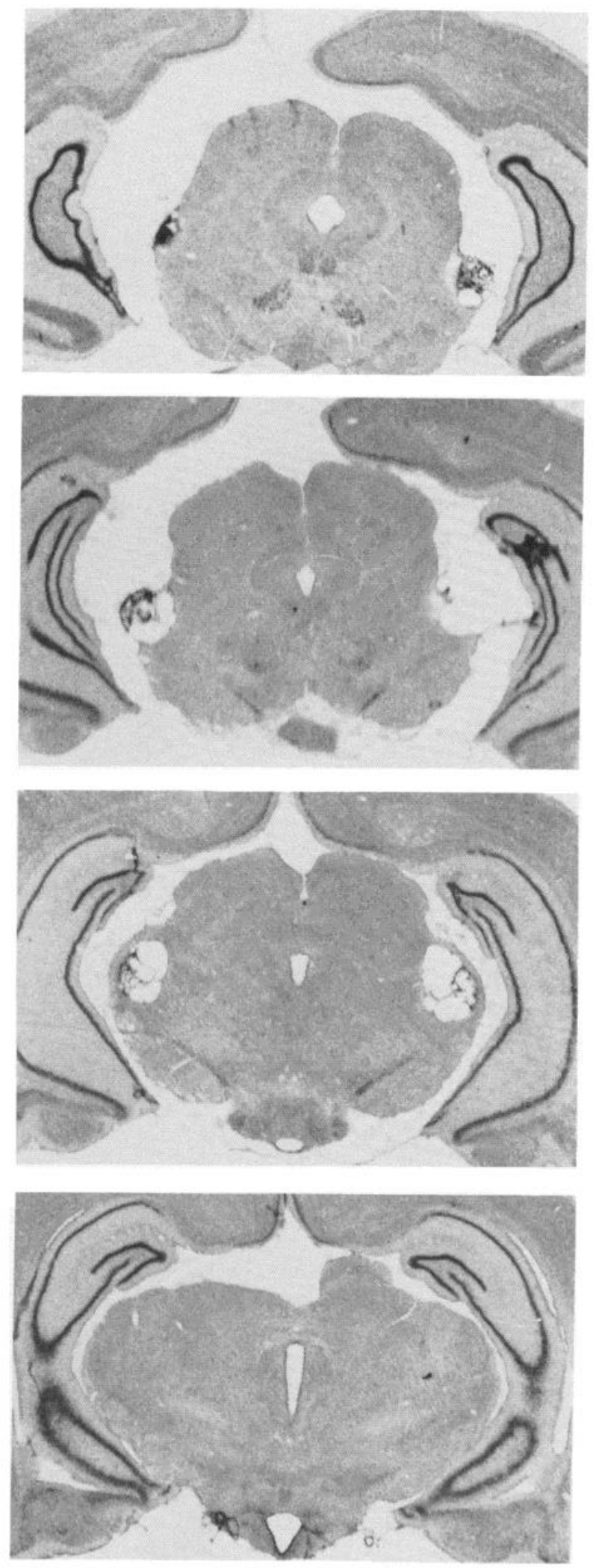

b
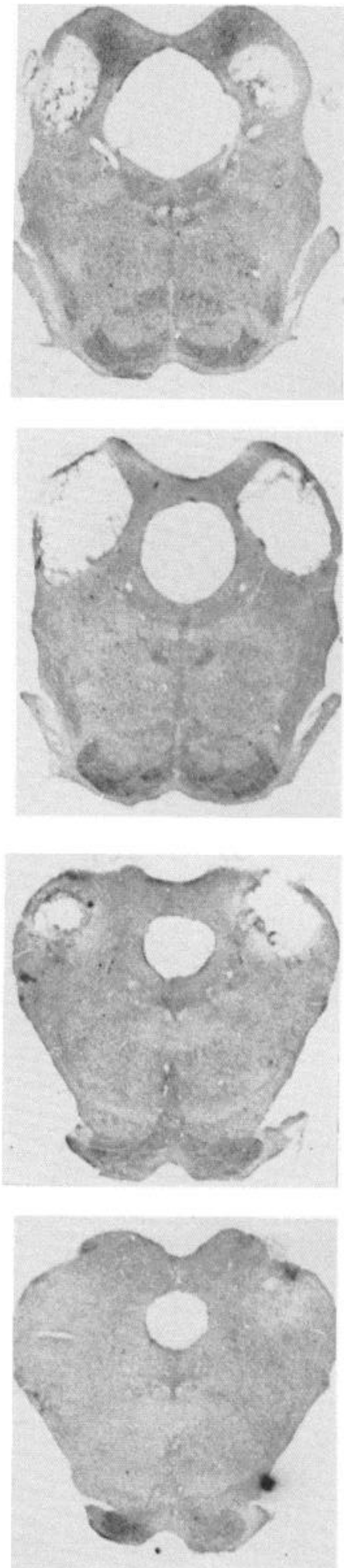

Figure 9. Photographs of cresyl violet-stained coronal sections through the anterior-posterior extent of typical lesions of MG and IC. 

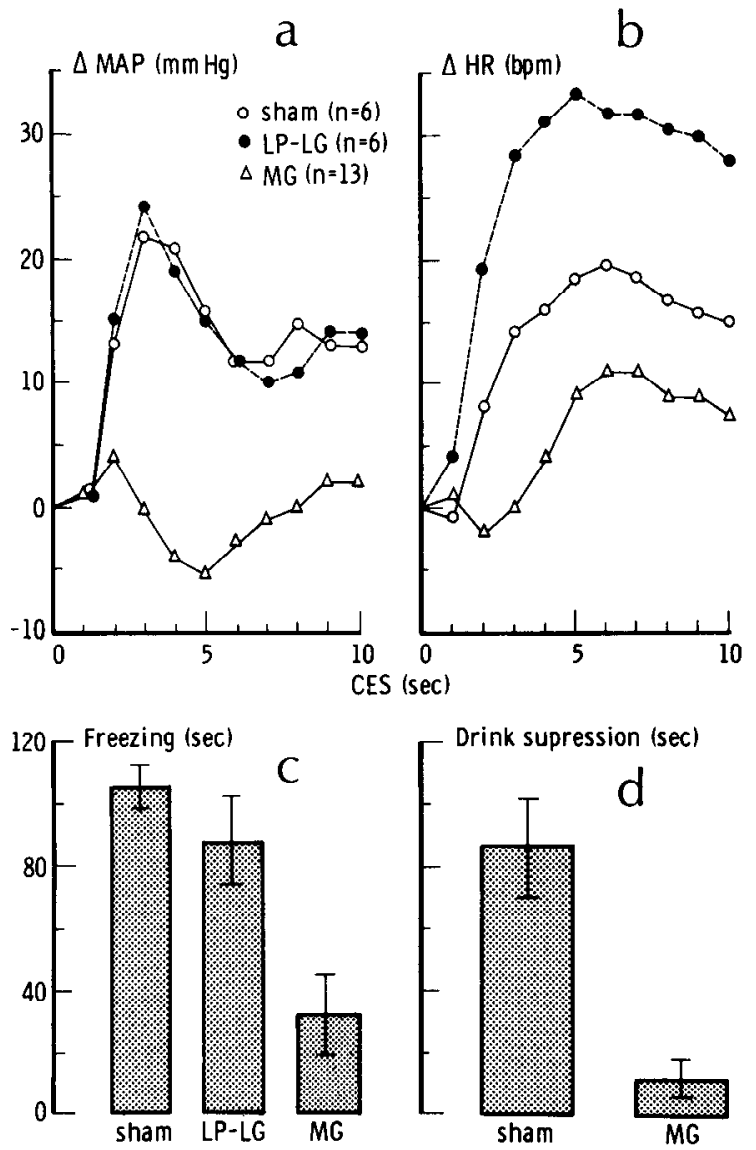

Figure 10. Effects of MG lesions on CERs. Lesions of MG significantly reduced the magnitude of the pressor response $(a)$ and tachycardia $(b)$ and the extent of freezing $(c)$ and drink suppression $(d)$ relative to sham-operated and LP-LG-lesioned rats. For the MAP and HR responses a two-way analysis of variance repeated measures design was employed, with treatment (MG versus LP-LG versus sham) as the fixed variable and every other second of the 10-sec CES as the repeated measure. The main effect of treatment was significant for the MAP response $(F(2,23)=21.0 ; p<0.01)$. Post-hoc evaluation using Dunnett's test indicated significant differences between MG and sham but not LP-LG and sham. The main effect of treatment was also significant for the HR response $(F(2,22)=$ $11.02 ; p<0.01)$. Dunnett's test indicated that both $\mathrm{MG}$ and LP-LG differed from sham controls, but only MG lesions impaired the response. The freezing response was evaluated using a one-way analysis of variance. The main effect of treatment was significant $(F(2,23)=32.76 ; p<0.01)$, and Dunnett's test indicated that only MG differed from sham. Drink suppression was evaluated using a $t$ test, which indicated that MG was impaired relative to sham controls $(t(11)=4.24 ; p<0.01)$. LPLG-lesioned rats were not tested for drink suppression.

(Fig. 7d) elicited by the CES. However, there was an increase in the variability of the cardiovascular responses. This variability did not appear to be related to lesion location or size, because the responses did not differ between the five animals with lesions inclusive of both the primary and secondary neocortical projection areas of MG and the 14 animals with lesions which destroyed the posterior but spared the anterior area (see above). All animals showed acoustic startle reactions to a hand clap.

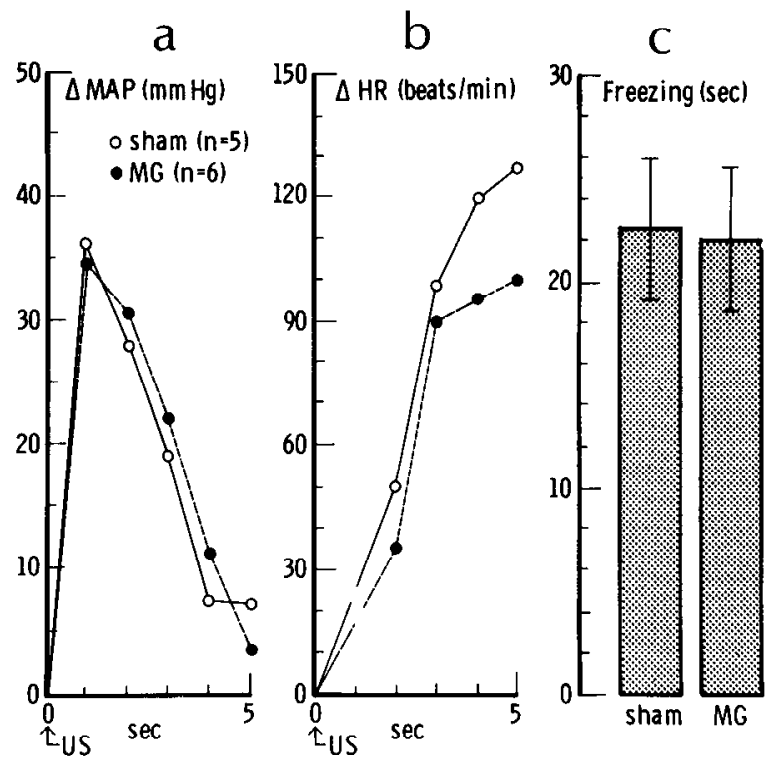

Figure 11. Effects of MG lesions on unconditioned responses. Lesions of $\mathrm{MG}$ did not affect the magnitude of the pressor response $(a)$ or tachycardia $(b)$ or the extent of freezing $(c)$ elicited by the footshock unconditioned stimulus. MAP and HR responses were analyzed using two-way analysis of variance repeated measures designs with treatment (lesion versus sham) as the fixed variable and each of the 5-sec intervals following the US as the repeated measure. Neither main effect was significant (MAP: $F(1,9)=0.33$; HR: $F(1,9)=2.6)$. Freezing was analyzed using a $t$ test, which was not significant $(t(10)=$ $0.13)$.

\section{Role of $M G$}

Lesions of $M G$. Bilateral electrolytic lesions were placed in MG in 13 rats (Fig. 8a). The lesions invariably destroyed the posterior two-thirds of the nucleus. The brachium of the inferior colliculus was also destroyed in all cases, but the inferior colliculus itself was not damaged (see Fig. 9). Damage to the anterior third of $\mathrm{MG}$ was variable from case to case, as was the medial extension of the lesion into the midbrain and thalamus. Lesions were larger in cases 9 to 13 than in cases 1 to 8 (Fig. 8a). Serial scetions through one of the lesions are shown in Figure 9.

Control lesions in the dorsal thalamus resulted in extensive damage in the region of the LP-LG (Fig. $8 b$ ). Lesion size was comparable to that in the animals with smaller lesions of MG (cases 1 to 8; Fig. 8a).

Effects of MG lesions on CERs. MG sham controls ( $n$ $=7$ ), like auditory cortex controls, exhibited a pressor response and tachycardia in response to the CES (Fig. $10, a$ and $b$ ). Moreover, the magnitude and patterning of the responses were similar in the two control groups. The extent of conditioned freezing (Fig. 10c) and drink suppression (Fig. 10d) in the MG control group was also similar to that in the auditory cortex control group.

The pressor response elicited by CES presentation was largely eliminated in MG-lesioned rats $(n=13)$ (Fig. 10a). A small depressor response, which peaked at the 5th sec following CES onset, remained. Lesions of LPLG $(n=6)$ did not affect the pressor response.

Lesions of MG delayed the onset and reduced the 
magnitude of the HR acceleration in response to the CES relative to sham-operated controls (Fig. 10b). Lesions of LP-LG resulted in a facilitation of the increase in HR, relative to MG sham operates, in response to the CES.

The extent of CES-elicited freezing (Fig. 10c) and drink suppression (Fig. 10d) was substantially reduced in MG-lesioned rats. However, LP-LG lesions did not affect conditioned emotional behavior (Fig. 10c).

The effects of MG lesions on behavioral and autonomic responses did not depend on lesion size, as the cases with relatively small (cases 1 to 8 ) and large (cases 9 to 13) lesions did not differ in any way. Moreover, lesions confined primarily to $\mathrm{MG}$, with little involvement of adjacent regions (see Fig. 9), were as effective as lesions which spread into adjacent nuclei.

In spite of these effects, MG lesions did not interfere with acoustic startle reactions. This is consistent with the results of studies showing that acoustic startle is mediated by circuits contained within the brainstem (Davis et al., 1982).

Effects on unconditioned emotional responses. To assess whether MG damage interfered with the expression of unconditioned fear responses elicited by the painful US, shock-eliciting freezing, blood pressure, and HR responses were examined in sham- and MG-lesioned animals.

In response to a single presentation of the $2.0-\mathrm{mA}, 0.5$ sec footshock US, MG- and sham-lesioned animals exhibited similar increases in MAP (Fig. 11a) and HR (Fig. $11 b$ ). Moreover, the extent of shock-elicited freezing did not differ in the two groups (Fig. 11c). Histological evaluation of the lesions in these cases revealed damage to $M G$ comparable to that described above for MG lesions 1 to 8 (Fig. 8).

Comparison of effects of $M G$ and IC lesions on CERs. If the effects of MG lesions on conditioned responses are due to damage to the ascending auditory pathway, damage to the immediately preceding auditory station, IC, should replicate the effects of MG damage. As shown in Figure 12, conditioned responses are similarly affected by damage to IC and MG. Serial sections through an IC lesion are shown in Figure 9.

\section{Discussion}

The purpose of the present study was to determine the role of the rodent auditory cortex and MG in the processing of an auditory CES. The rat auditory cortex was identified through neuroanatomical tracing studies in volving the anterograde transport of HRP microinjected into MG. The effects of auditory cortex and MG lesions on autonomic and behavioral responses coupled through classical fear conditioning to an auditory stimulus were examined. The results demonstrate that lesions of $\mathrm{MG}$, but not of the auditory cortex, disrupt the development and expression of both autonomic and behavioral emotional responses coupled to an auditory CES.

Cortical and subcortical efferent projections of $M G$. The geniculocortical link in the auditory system has been extensively studied in cats and primates (for review see Rose and Woolsey, 1958; Harrison and Howe, 1974; Casseday et al., 1976; Oliver and Hall, 1978), but rela- tively little work has been done in rats. Studies examining retrograde cell changes in the thalamus following cortical lesions provide the primary existing source of data concerning the rat auditory cortex (Waller, 1934; Lashley, 1941; Krieg, 1946; Kelly and Glazier, 1978). While several studies have evaluated nerve terminals in the rodent neocortex following MG lesions using silver degeneration methods (Ryugo and Killackey, 1974; Vaughan, 1983), these were not aimed at mapping the efferent projection zone of MG.

Microinjection of HRP into MG resulted in the anterograde labeling of two neocortical terminal zones. Whether these represent two independent projections cannot be determined at present. However, in serial sections the two areas appeared discontinuous.

The primary (posterior) zone encompasses the region identified by Krieg as the auditory cortex (area 41), together with areas 39 and 40 , and portions of areas 2 and 2a (Krieg, 1946). This posterior zone corresponds almost exactly to the region from which auditory evoked responses can be recorded in rat (LeMessurier, 1948; Woolsey and LeMessurier, 1948; Woolsey, 1952). These data are in close agreement with a recent study by Guldin and Markowitsch (1983), who found retrograde transport of HRP to MG when lateral cortical injections aimed at the insular cortex were placed superior to the rhinal fissure in the region we identified as the posterior projections zone of $\mathrm{MG}$.

The secondary (anterior) zone is located in the anteroventral aspect of Krieg's area 2, just anterior to area 2a. While this region, which lies in the somatosensory cortex and which has not previously been identified as a target of MG efferents, may be a secondary auditory region, it is also possible that the terminals originate in the multimodal, medial nucleus of MG (Nauta and Kuypers, 1958; Lund and Webster, 1967; Oliver and Hall, 1978; Blum et al., 1979) and subserve somatosensation. Alternatively, the anterior region might reflect uptake of HRP by damaged axons coursing through MG and projecting to the neocortex.

Subcortical efferent projections of MG included several areas outside of the primary auditory system: caudate-putamen, central and lateral nuclei of the amygdala, ventromedial hypothalamus, and the subparafascicular region of the thalamus. Although we have not yet conducted retrograde experiments to confirm that these projections arise in MG, published retrograde studies have demonstrated afferents to the amygdala from the medial nucleus of MG (Veening, 1978; Ottersen and BenAri, 1979). Moreover, select lesions of the medial MG result in terminal degeneration in the caudate-putamen (Ryugo and Killackey, 1974). Thus, results obtained by others using different techniques confirm the existence of projections from MG to amygdalar and striatal areas and substantially weaken the possibility that our observations are due to the spread of HRP to areas adjacent to MG or to the uptake of $\mathrm{HRP}$ by fibers coursing through MG. Whether the hypothalamic and subparafascicular projections also arise in subnuclei of MG or are instead accounted for by HRP diffusion or by uptake by fibers of passage is not yet known.

In summary, anterograde HRP experiments were used 

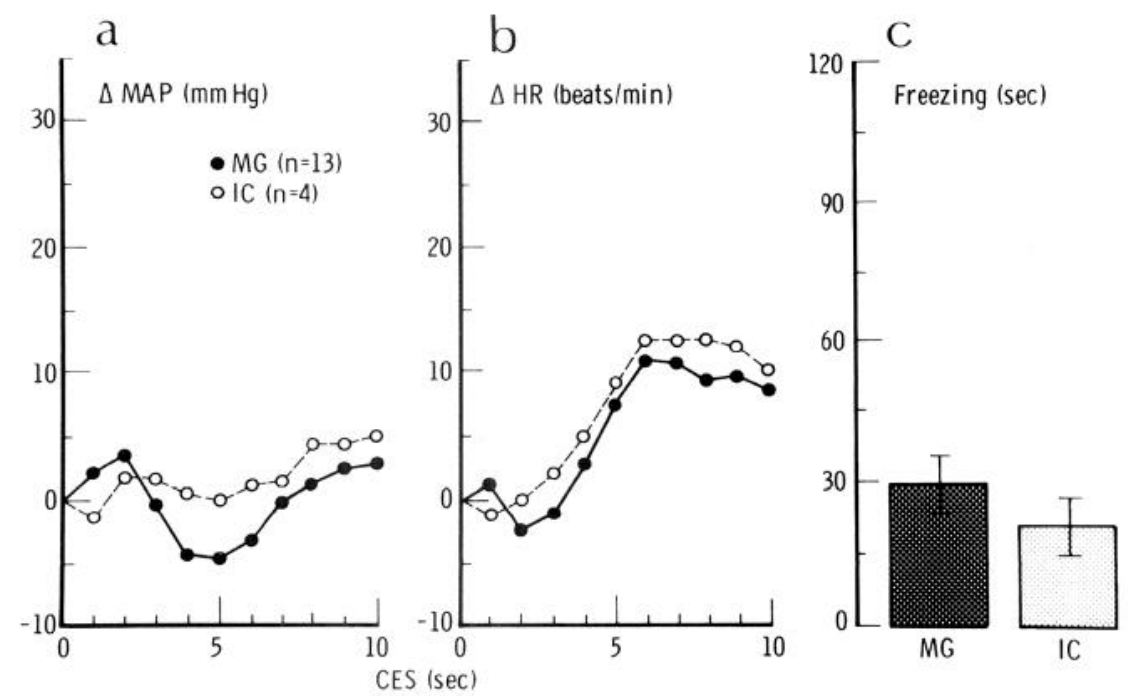

Figure 12. Comparison of the effects of MG and IC lesions. Animals with lesions of MG and IC performed identically in all tests. Thus, lesions of IC completely replicated the effects of $\mathrm{MG}$ lesions on autonomic and behavioral CERs.

to identify the auditory cortex in rat. However, projections from MG to subcortical areas were also observed. These subcortical projections are of particular interest since several of the target regions (amygdala, caudate, hypothalamus) have been implicated in the control of emotional behavior and autonomic function (see Issacson, 1982).

Neocortex and the conditioning of emotional responses. To evaluate the effects of auditory cortex lesions on the processing of an acoustic CES, lateral neocortical regions were ablated, and the extent of damage was histologically compared with the distribution of neocortical efferents from MG. Some lesions included both the primary and secondary MG projection areas, whereas others only included the secondary region. Neither behavioral nor autonomic responses to the acoustic CES were significantly impaired in either lesion subgroup.

These results are consistent with previous studies showing that complete neodecortication, which necessarily destroys the auditory neocortex, does not prevent the establishment of cardiac conditioned responses in rats (Bloch-Rojas et al., 1964; DiCara et al., 1970). At odds with the present results is the earlier observation that behavioral conditioned responses are impaired in totally decorticated rats (Pinto-Hamuy et al., 1963; Bloch-Rojas et al., 1964). However, Kelly and Glazier (1978) have found that lateral neocortical ablations which result in extensive retrograde cell changes in MG do not impair discriminated conditioned suppression of auditory spatial cues in rats. Together with the present results, Kelly and Glazier's observation indicates that the possible effects of complete decortication on behavioral conditioned responses are not due to a disruption of the neocortical auditory system. This conclusion is in agreement with many studies in cats and primates which have shown that auditory cortex ablations do not impair behavioral responses based on sound detection, auditory sensitivity, frequency discrimination, or intensity discrimination (see Neff et al., 1975; Neff and Diamond, 1958; Masterson and Diamond, 1978).
In summary, autonomic and behavioral responses elicited by an undiscriminated acoustic CES survive auditory cortex ablations in rats. Thus, the processing of the emotional significance of such stimuli is based on afferent information diverging from the auditory pathway prior to arriving at the neocortex. Therefore, while cortical sensory receiving areas are part of the circuitry underlying emotional responses based on complex perceptual discriminations (see the introduction), emotional responses coupled to relatively simple stimulus features are mediated by pathways which bypass the neocortical sensory systems.

$M G$-Auditory afferent link in emotional processing. Lesions of MG, the auditory thalamic relay nucleus, abolished the conditioned pressor response and conditioned freezing and drink suppression. Thus, these CERs appear to depend upon functions represented in or near MG.

The effects of MG damage on autonomic and somatomotor emotional responses are most likely due to a disruption of ascending auditory input. This view is supported by the fact that lesions of IC, the primary source of afferent input to MG, totally replicated the effects of MG lesions. Moreover, since lesions of auditory structures efferent to MG (i.e., the cortical auditory areas) did not interfere with auditory emotional conditioning, we conclude that afferent information diverging from the ascending auditory pathway at the level of MG sustains the development of emotional responses to acoustic stimuli.

HR responses, although impaired by MG and IC damage, were not affected to the same extent as the behavioral and pressor responses. This suggests that the HR response can be sustained, at least in part, by auditory centers below MG and IC.

The fact that lesions of MG did not alter behavioral or autonomic responsivity to footshock eliminates the possibility that the effects on the conditioned responses were due to changes in unconditioned emotional reactivity or to a disruption of the efferent pathways underlying 
these responses. Since MG-lesioned animals exhibited acoustic startle responses, which are mediated by circuits within the brainstem (Davis et al., 1982), the results cannot be attributed to peripheral deafness or to disruption of brainstem auditory functions.

Our results do not distinguish between effects of $\mathrm{MG}$ lesions on the acquisition and expression of the emotional responses. This is an important point to consider since a number of studies have shown that unit activity in MG is modified during auditory conditioning (Olds et al., 1972; Disterhoft and Stuart, 1976; Gabriel et al., 1976; Ryugo and Weinberger, 1978; Birt et al., 1979). We assume that lesions placed after conditioning and prior to testing would have effects similar to those reported here, but additional studies will be required to determine whether this is so.

Although MG is composed of several structural and functional subdivisions (see Harison and Howe, 1974), certain observations are consistent with the view that the medial division of $M G$ is the source of afferent information underlying auditory emotional conditioning. First, the medial nucleus, like other divisions, receives direct input from IC (J. E. LeDoux, unpublished observation; Moore and Goldberg, 1963; Casseday et al., 1976). Second, following removal of the auditory cortex, neurons in the ventral nucleus of MG, the classic thalamocortical relay nucleus, degenerate, but neurons in the medial nucleus survive (see "Results"). Third, as described, the medial but not the ventral nucleus projects to limbic and striatal area implicated in emotional behavior and autonomic regulation.

In summary, we have, using anatomical tracing and behavioral techniques, begun to identify the neural pathway through which the emotional meaning of environmental events is evaluated. The results indicate that for relatively simple auditory stimuli in rats the evaluation is based on afferent information diverging from the primary sensory projection pathway subcortically, at the level of the MG. Additional studies will be required to determine the theoretical implications of this demonstration that sensory information can exert meaningful influences over autonomic function and behavior through subcortical neural pathways.

\section{References}

Bard, P., and D. M. Rioch (1937) A study of four cats deprived of neocortex and additional portions of the forebrain. Bull. Johns Hopkins Hosp. 60: 73-125.

Bechterev, W. (1887) Die bedentung der sehhujel auf grand von experimentellen und pathologischen daten. Virchow's Arch. 110: 302-322.

Birt, D., R. Nienhuis, and M. Olds (1979) Separation of associative from nonassociative short latency changes in medial geniculate and inferior colliculus during differential conditioning and reversal in rats. Brain Res. 167: 129-138.

Blanchard, D. C., and R. J. Blanchard (1972) Innate and conditioned reactions to threat in rats with amygdaloid lesions. J. Comp. Physiol. Psychol. 81: 281-290.

Bloch-Rojas, S., A. Toro, and T. Pinto-Hamuy (1964) Cardiac and somatomotor conditioned responses in neodecorticate rats. J. Comp. Physiol. Psychol. 58: 233-239.

Blum, P. S., L. D. Abraham, and S. Gilman (1979) Vestibular, auditory and somatic input to the posterior thalamus of the cat. Exp. Brain Res. 34: 1-9.
Bouton, M. E., and R. C. Bolles (1980) Conditioned fear assessed by freezing and by the suppression of three different baselines. Animal Learning Behav. 8: 429-434.

Bromiley, R. B. (1948) Conditioned responses in a dog after removal of neocortex. J. Comp. Physiol. Psychol. 41: 102110.

Cannon, W. B., and S. W. Britton (1925) Pseudo-affective medulliadrenal secretion. Am. J. Physiol. 72: 283-294.

Casseday, J. H., I. T. Diamond, and J. K. Harting (1976) Auditory pathways to the cortex in Tupeia glis. J. Comp. Neurol. 166: 303-340.

Culler, E., and F. A. Mettler (1934) Conditioned behavior in a decorticate dog. J. Comp. Physiol. Psychol. 18: 291-303.

Davis, M., D. S. Gendelman, M. D. Tischler, and P. M. Gendleman (1982) A primary acoustic startle circuit: Lesion and stimulation studies. J. Neurosci. 2: 791-805.

Diamond, I. T., and W.D. Neff (1957) Ablation of temporal cortex and discrimination of auditory patterns. J. Neurophysiol. 20: 300-315.

DiCara, L., J. J. Braun, and B. Pappas (1970) Classical conditioning and instrumental learning of cardiac and gastrointestinal responses following removal of neocortex in the rat. $J$. Comp. Physiol. Psychol. 73: 208-216.

Disterhoft, J., and D. Stuart (1976) Trial sequence of changed unit activity in auditory system of alert rat during conditioned response acquisition and extinction. J. Neurophysiol. 3.9: $266-281$

Downer, J. D. C. (1961) Changes in visual gnostic function and emotional behavior following unilateral temporal lobe damage in the "split-brain" monkey. Nature (Lond.) 191: 50-51.

Gabriel, M., S. E. Slatwick, and J. D. Miller (1976) Multiple unit activity of the rabbit medial geniculate nucleus in conditioning, extinction and reversal. Physiol. Psychol. 4: 124134.

Geschwind N. (1965) The disconnexion syndromes in animals and man. Brain 88: 237-294.

Girden, E., F. A. Mettler, G. Finch, and E. Culler (1936) Conditioned responses in a decorticate dog to acoustic, thermal and tactile stimulation. J. Comp. Physiol. Psychol. 21: 367-385.

Graham, R. C., Jr., and M. J. Karnovsky (1966) The early stages of absorption of horseradish peroxidase in the proximal tubules of mouse kidney: Ultrastructural cytochemistry by a new technique. J. Histochem. Cytochem. 14: 291-302.

Gray, J. A. (1982) The Neuropsychology of Anxiety, Oxford University Press, Oxford.

Guldin, W. O., and J. Markowitch (1983) Cortical and thalamic afferent connections of the insular and adjacent cortex of the rat. J. Comp. Neurol. 215: 135-153.

Harrison, J. M., and M. E. Howe (1974) Anatomy of the afferent auditory nervous system in mammals. In Handbook of Sensory Physiology, W. D. Keidel and W. D. Neff, eds., Vol. V/ 1, pp. 283-336, Spring-Verlag, Berlin.

Herzog, A. G., and G. W. van Hoesen (1976) Temporal neocortical afferent connections to the amygdala in the rhesus monkey. Brain Res. 115: 57-69.

Horel, J. A., and E. G. Keating (1969) Partial Kluver-Bucy syndrome produced by cortical disconnection. Brain Res. 16 : 281-284.

Issacson, R. L. (1982) The Limbic System, Plenum Press, New York.

Jones, B., and M. Mishkin (1972) Limbic lesions and the problem of stimulus-reinforcement associations. Exp. Neurol. 36: 362-377.

Jones, E. G., and R. Y. Leavitt (1974) Retrograde axonal transport and the demonstration of non-specific projections to the central cortex and striatum from thalamic intralaminar nuclei in the rat, cat, and monkey. J. Comp. Neurol. 154: 349-378. 
Jones, E. G., and T. P. S. Powell (1970) An experimental study of converging sensory pathways within the cerebral cortex of the monkey. Brain 93: 793-820.

Kelly, J. B. (1974) Polysensory cortical lesions and auditory temporal pattern discrimination in the cat. Brain Res. 80 : $317-327$.

Kelly, J. B., and S. J. Glazier (1978) Auditory cortex lesions and discrimination of spatial location by the rat. Brain Res. 145: 315-321.

Krieg, W. S. J. (1946) Connections of the cerebral cortex in the albino rat. A topography of the cortical areas. J. Comp. Neurol. 84: 221-275.

Lashley, K. S. (1941) Thalamo-cortical connections of the rat's brain. J. Comp. Neurol. 75: 67-121.

Leaf, R. C., and S. A. Muller (1965) Simple method for CER conditioning and measurement. Psychol. Rep. 16: 211-215.

LeDoux, J. E. (1984) Cognition and emotion: Processing functions and neural systems. In Handbook of Cognitive Neuroscience, M. S. Gazzaniga, ed., Plenum Press, New York.

LeDoux, J. E., A. Sakaguchi, and D. J. Reis (1982) Behaviorally-selective cardiovascular hyperreactivity in spontaneously hypertensive rats: Evidence for hypoemotionality and enhanced appetitive motivation. Hypertension 4: 853863.

LeDoux, J. E., A. Sakaguchi, and D. J. Reis (1983) Strain difference in fear between spontaneously hypertensive and normotensive rats. Brain Res. 227: 137-143.

LeMessurier, D. H. (1948) Auditory and visual areas of the cerebral cortex of rat. Fed. Proc. 7: 70.

Lund, R. D., and K. E. Webster (1967) Thalamic afferents from the dorsal column nuclei. An experimental study in rat. J. Comp. Neurol. 130: 310-312.

MacLean, P. D. (1949) Psychosomatic disease and the visceral brain. Recent developments bearing on the Papez theory of emotion. Psychosom. Med. 11: 338-353.

MacLean, P. D. (1970) The triune brain, emotion and scientific bias. The Neurosciences: Second Study Program, F. 0. Schmitt, ed., pp. 336-348, Rockefeller University Press, New York.

Macht, M. D., and P. Bard (1942) Studies on decerebrate cats in the chronic state. Fed. Proc. 1: 55-56.

Masterson, B., and I. T. Diamond (1978) Hearing. Central neural mechanisms. In Handbook of Perception, E. C. Carterette and M. P. Friedman, eds., pp. 408-448, Academic Press, Inc., New York.

Mesulam, M. -M. (1978) Tetramethylbenzidine for horseradish peroxidase neurohistochemistry: A non-carcinogenic blue reaction product with superior sensitivity for visualizing neural afferents and efferents. J. Histochem. Cytochem. 26: 106117.

Mesulam, M. -M. (1982) Tracing Neural Connections with Horseradish Peroxidase, John Wiley \& Sons, New York.

Meyer, D. R., and C. N. Woolsey (1952) Effect of localized cortical destruction on auditory discriminative conditioning in cat. J. Neurophysiol. 15: 149-162.

Mishkin, M. (1956) Visual discrimination performance following partial ablations of the temporal lobe. II. Ventral surface vs. hippocampus. J. Comp. Physiol. Psychol. 47: 187-193.

Mishkin, M. (1978) Memory in monkeys severely impaired by combined but not separate removal of amygdala and hippocampus. Nature 273: 297-298.

Moore, R. Y., and J. M. Goldberg (1963) Ascending projections of the inferior colliculus in the cat. J. Comp. Neurol. 121: 109-136.

Moreland, R. L., and R. B. Zajonc (1979) Exposure effects may not depend on stimulus recognition. J. Personality Social Psychol. 37: 1085-1089.

Morest, D. K. (1964) The neuronal architecture of the medial geniculate body of the cat. J. Anat. (Lond.) 98: 611-630.
Myers, R. D. (1971) Methods in Psychobiology, Vol. 1, Academic Press, Inc., New York.

Nauta, W. J., and H. G. J. M. Kuypers (1958) Some ascending pathways in the brainstem reticular formation. In Reticular Formation of the Brain, J. J. Jasper, ed., pp. 3-30, Little, Brown, Boston.

Neff, W. D., and I. T. Diamond (1958) The neural basis of auditory discrimination. In The Biological and Biochemical Basis of Behavior, H. F. Harlow and C. N. Woolsey, eds., pp. 101-126, University of Wisconsin Press, Madison.

Neff, W. D., I. T. Diamond, and J. H. Casseday (1975) Behavioral studies of auditory discrimination: Central nervous system. In Handbook of Sensory Physiology, W. D. Keidel and W. D. Neff, eds., pp. 307-400, Springer-Verlag, Heidelberg.

Olds, J., J. F. Disterhoft, M. Segal, C. Kornbluth, and R. Hirsh (1972) Learning centers of rat brain mapped by measuring latencies of conditioned unit responses. J. Neurophysiol. 35: 202-219.

Oliver, D. J., and W. C. Hall (1978) The medial geniculate body of the tree shrew, Tupeia glis. II. Connections with neocortex. J. Comp. Neurol. 182: 459-494.

Ottersen, O. P., and Y. Ben-Ari (1979) Afferent connections to the amygdaloid complex of the rat and cat. J. Comp. Neurol. 187: 401-424.

Papez, J. W. (1937) A proposed mechanism of emotion. Arch. Neurol. Psychiatry 79: 217-224.

Paxinos, G., and C. Watson (1982) The Rat Brain in Stereotaxic Coordinates, Academic Press, Sydney.

Penfield, W. (1958) Centrencephalic integrating system. Brain 81: 231-234.

Pinto-Hamuy, T., H. G. Santibanez, and J. A. Rojas (1963) Learning and retention of a visual conditioned response in neodecorticate rats. J. Comp. Physiol. Psychol. 56: 19-24.

Rose, J. E., and C. N. Woolsey (1958) Cortical connections and functional organization of thalamic auditory system of cat. In Biological and Biochemical Bases of Behavior, H. F. Harlow and C. N. Woolsey, eds., pp. 127-150, University of Wisconsin Press, Madison.

Ruggiero, D. A., C. A. Ross, M. Kumada, and D. J. Reis (1982) Reevaluation of projections from the mesencephalic trigeminal nucleus to the medulla and spinal cord: A combined retrograde and anterograde horseradish peroxidase study. $\mathrm{J}$. Comp. Neurol. 206: 278-292.

Ryugo, D. K., and H. P. Killackey (1974) Differential telencephalic projections of ventral and medial division of the medial geniculate body of the rat. Brain Res. 82: 173-177.

Ryugo, D. K., and N. M. Weinberger (1978) Differential plasticity of morphologically distinct neuron populations in the medial geniculate body of the cat during classical conditioning. Behav. Biol. 22: 275-301.

Sakaguchi, A., J. E. LeDoux, and D. J. Reis (1983) Sympathetic nerves and adrenal medulla: Differential contributions to cardiovascular-conditioned emotional responses in spontaneously hypertensive rats. Hypertension 5: 728-738.

Skinner, J. E. (1971) Neuroscience: A Laboratory Manual, W. B. Saunders, Philadelphia.

Thompson, R. F. (1960) Function of auditory cortex of cat in frequency discrimination. J. Neurophysiol. 23: 321-334.

Turner, B. H., M. Mishkin, and M. Knapp (1980) Organization of the amygdalopetal projections from modality-specific cortical association areas in the monkey. J. Comp. Neurol. 191: $515-543$.

van Hoesen, G. W., D. N. Pandya, and N. Butters (1972) Cortical afferents to the entorhinal cortex of the rhesus monkey. Science 175: 1471-1473.

Vaughan, D. (1983) Thalamic and callosal connections of the rat auditory cortex. Brain Res. 260: 181-189.

Veening, J. G. (1978) Subcortical afferents of the amygdaloid 
complex in the rat: An HRP study. Neurosci. Lett. 8: 197202.

Waller, W. H. (1934) Topographical relations of cortical lesions to thalamic nuclei in the albino rat. J. Comp. Neurol. 60: 237-270.

Whitlock, D. G., and W. J. H. Nauta (1956) Subcortical projections from the temporal neocortex in Macaca mulatla. J. Comp. Neurol. 106: 183-212.
Woolsey, C. N. (1952) Patterns of localization in sensory and motor areas of the cerebral cortex. In Biology of Mental Health and Disease, pp. 193-206, Hoeber, New York.

Woolsey, C. N., and D. H. LeMessurier (1948) The pattern of cutaneous presentation in the rat's cerebral cortex. Fed. Proc. 7: 137.

Zajonc, R. B. (1980) Feeling and thinking: Preferences need no inferences. Am. Psychol. 35: 151-175. 